\section{Detection and Recording of Optical Transients by Photo-multipliers}

THE use of photo-multipliers for the detection and recording of optical transients is well known in the fields of gaseous discharge and of scintillation counting. In both these fields, involving timeintervals short compared with a microsecond, the limit of detection of the system is set by the shot noise associated with the photo-current and not by the thermionic emission from the cathode. The thermionic emission from the cathode of a photomultiplier is usually quoted as about $10^{-15} \mathrm{amp} . / \mathrm{cm} .^{2}$ or approximately $10^{-14}$ amp. for the whole cathode. This corresponds to an average rate of emission of electrons of approximately one electron in ten microseconds, and the probability of emission of an electron during any interval of time short compared with a microsecond is small.

The shot noise associated with a photo-current $I_{0}$ is given by :

$$
\left(i_{n}\right)_{\mathrm{rms}}=\left(2 e I_{0} . \delta f\right)^{1 / 2},
$$

where $\delta f$ is the bandwidth of the system and $e$ is the electronic charge.

If we take the limit of detection to be :

$$
\begin{gathered}
\left(i_{n}\right)_{\mathrm{rms}}=I_{0}, \\
I_{0}=2 e . \delta f .
\end{gathered}
$$

then

If the output current of the photo-multiplier, $g I_{0}$, where $g$ is the gain, is being used to produce a voltage across an anode resistor $R$ in parallel with the anode and stray capacitance $C$, then we may write approximately

$$
\text { and therefore } \quad I=g e / C R \text {, }
$$

where $I$ is the output current of the photo-multiplier.

The output voltage generated across the anode resistor $R$ is :

$$
V=R I=g e / C .
$$

From equation (6) we see that this minimum value of $V$ is independent of the value of $R$, varies directly as the gain of the photo-multiplier and is inversely proportional to the capacitance $C$. In the applications quoted above, the amount of light available is usually not much greater than the minimum amount that can be detected, and a certain amount of amplification of the resulting signals is needed. By inserting values of $g$ and $C$ in equation (6), the minimum voltage which the amplifier will be required to amplify may be determined. The output capacitance of an R.C.A. 931-A type of photo-multiplier is approximately $5 \mathrm{pF}$., and therefore a value of $10 \mathrm{pF}$. may be taken for $C$. The maximum gain obtainable from such a photo-multiplier is around $10^{8}$, and therefore the minimum value of $V$ for detection will be 0.016 volt.

From equation (5) we see that the minimum photocurrent for detection corresponds to the release of one photo-electron in a time of $C R$. Since the efficiency of the photo-cathode of the 931-A type of photomultiplier is such that approximately ten incident quanta are required to release one photo-electron, the minimum detectable light flux will correspond to the incidence of approximately ten quanta on to the cathode in a time of $C R$.

The above analysis of the minimum detectable signals neglects the contribution of the secondary emission to the noise-level observed at the anode of the photo-multiplier, as this has been shown to be small. It also assumes that the thermal agitation noise in the resistor $R$ produces a noise voltage which is small compared with the amplified shot noise $g I_{0}$. The thermal agitation noise in a resistor is given by :

$$
\left(v_{n}\right)_{\mathrm{rms}}=(4 k T R \delta f)^{1 / 2}
$$

Substituting for $\delta f$ from equation (4), we have :

$$
\left(v_{n}\right)_{\mathrm{rms}}=(2 k T / C)^{1 / 2}
$$

In the limit, when the noise due to the resistor is equal to the amplified shot noise,

$$
g=\left(2 k T C / e^{2}\right)^{1 / 2} .
$$

The above value of $g$ represents the minimum gain which may be used to ensure that the noise across the resistor $R$ is not the limiting factor in the detection of small values of light flux. It will be seen that this minimum value of gain is independent of the value of $R$ but depends on the value of $C$. For a value of $C$ of $10 \mathrm{pF}$., $g_{\min }$ is 2,500 .

The above analysis has been based on the detection of a light flux, and a similar analysis may be performed where the object is the measurement of a light flux to an accuracy of one part in $N$. To attain this accuracy, the minimum number of photoelectrons which must leave the photo-cathode in the minimum resolution time $\tau$ is $N^{2}$ and therefore the

\begin{tabular}{|c|c|c|c|}
\hline \multirow{2}{*}{ Gain } & \multicolumn{3}{|c|}{ Resolution time } \\
\hline & $10^{-9}$ sec. & $10^{-8} \mathrm{sec}$ & $10^{-7}$ sec. \\
\hline $\begin{array}{l}10^{4} \\
10^{5} \\
10^{3} \\
10^{7} \\
10^{8}\end{array}$ & $\begin{array}{c}160 \text { mamp. } \\
1.6 \text { m.amp. } \\
16 \text { m.amp. } \\
160 \text { m.amp. } \\
1.6 \text { amp. }\end{array}$ & $\begin{aligned} 16 & \mu \text { amp. } \\
160 & \mu \text { amp. } \\
1 \cdot 6 & \text { m.amp. } \\
16 & \text { m.amp. } \\
160 & \text { m.amp. }\end{aligned}$ & 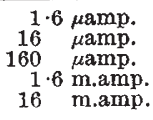 \\
\hline
\end{tabular}
minimum output current is :

$$
I_{\min }=N^{2} g e / \tau \text {. }
$$

\begin{tabular}{|c|c|c|c|}
\hline \multirow{2}{*}{ Gain } & \multicolumn{3}{|c|}{ Resolution time } \\
\hline & $10^{-9} \mathrm{sec}$. & $10^{-8}$ sec. & $10^{-7}$ sec. \\
\hline $\begin{array}{l}10^{4} \\
10^{5} \\
10^{\mathrm{s}} \\
10^{7} \\
10^{8}\end{array}$ & $\begin{array}{c}16 \text { m.amp. } \\
160 \text { m.amp. } \\
1.6 \text { amp. } \\
16 \text { amp. } \\
160 \text { amp. }\end{array}$ & $\begin{array}{c}1.6 \text { m.amp. } \\
16 \text { m.amp. } \\
160 \text { m.amp. } \\
1.6 \text { amp. } \\
16 \text { amp. }\end{array}$ & 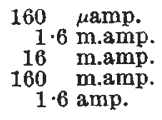 \\
\hline
\end{tabular}

Tables 1 and 2 show the minimum output currents required to maintain accuracies of 10 and 1 per cent respectively.

Table 1. Mrnimum Odtput CoRrents for Accuracies of 10 Per Cext

Table 2. Minimum OUtput CuRRents for ACcuracies of 1 Per Cest

The use of the R.C.A. 931-A type of photomultiplier enables currents of up to about $80 \mathrm{~m} . \mathrm{amp}$. to be obtained with a resolution time of around $1 \times 10^{-8}$ sec., and therefore the maximum gain which may be used to obtain an accuracy of 1 per cent is approximately $10^{5}$. A gain of $10^{7}$ lowers the accuracy which can be obtained to 10 per cent.

The above analysis shows that it is as important to endeavour to increase the maximum linear current which can be drawn from a photo-multiplier as to endeavour to increase the maximum gain which can be obtained.

I acknowledge the grant of an I.C.I. Research Fellowship which made this work possible.

Department of Electrical Engineering, R. F. SAXE Queen Mary College,

University of London. Aug. 12. 